\title{
A prospective cohort study on physical activity and sports-related injuries in 10-12-year-old children
}

\author{
E Verhagen, D Collard, M Chin A Paw, W van Mechelen
}

Department of Public and Occupational Health, EMGO Institute, VU University Medical Center, Amsterdam, The Netherlands

Correspondence to: E Verhagen, Department of Public and Occupational Health, EMGO Institute, VU University Medical Center, Van der Boechorststraat 7, 1081 BT Amsterdam, The Netherlands; e.verhagen@vumc.nl

Accepted 13 December 2008 Published Online First

5 January 2009

\begin{abstract}
Objectives: To describe the incidence and severity of injuries resulting from physical education, sports and leisure time physical activity (PA) in 10-12-year-old children.
\end{abstract}

Design: This was a prospective cohort study conducted in primary schools, with 995 children aged 10-12 years old as participants. Individual weekly exposure was estimated from baseline and follow-up questionnaires. Exposure to physical education (PE) classes was equal in all schools. An injury was recorded if it occurred during either PE class, leisure time PA, or sports, and caused the child to at least stop the current activity. Injuries were reported within 1 week of injury onset. The main outcome measure was injury incidence density.

Results: During the school year a total of 119 injuries were reported by 104 children, resulting in an overall injury incidence density (ID) of 0.48 per $1000 \mathrm{~h}$ of exposure (95\% Cl 0.38 to 0.57). Injury ID was lowest for leisure time PA, followed by PE and sports, respectively. Of all injuries, 40\% required medical treatment and 14\% resulted in 1 or more days of absence from regular school activities. In general for girls a higher injury ID was reported than for boys, mainly caused by a twofold higher risk during leisure time PA.

Conclusions: Next to specific areas of preventive interest it was found that in this specific age group, girls require special attention as they seem to be at higher injury risk than boys.

The numerous health benefits of regular physical activity (PA) in children are well described in the literature. ${ }^{12}$ With the subsequent focus on a physically active lifestyle, an increasing number of sports and physical activity injuries can be expected. ${ }^{3}{ }^{4}$ These injuries lead to high direct and indirect costs. ${ }^{5}$ More importantly, children may lose their enthusiasm for PA and sports through negative associations with injuries. ${ }^{6}$ Consequently, the successful prevention of sports and PA injuries in youth has great potential health gain. ${ }^{7}$ In the short term, the absolute number of injuries will fall and, in the longer term, the risk of injury recurrences and prolonged periods of impairment will be prevented. Prevention may also promote a physically active lifestyle from childhood into adulthood.

An important purpose of injury epidemiology is to supply knowledge on injuries that occur frequently and have serious consequences, and to describe their aetiology in order to provide a basis for preventive measures. ${ }^{8}$ A recent review on the risk of sports injuries in children revealed that epidemiological data on this topic is scarce. ${ }^{9}$ Moreover, it was concluded that there is a high need for more insight into injuries during unorganised activities and for younger age groups.

In particular, the combination of unorganised activities in younger age groups requires attention. An active child is growing, learning and developing its skills. During this process the younger child "evolves" from a participant in joyful PA to a participant in sports. ${ }^{7}$ Therefore, the purpose of this prospective cohort study was to describe the incidence and severity of injuries resulting from physical education (PE), sports and leisure time PA in 10-12-year-old Dutch children.

\section{METHODS}

\section{Population}

This study was part of the iPlay study, an injury prevention study in 10-12-year-old children that has been carried out in primary schools located throughout The Netherlands. ${ }^{10}$ From the 7000 primary schools throughout The Netherlands, 520 primary schools were randomly selected and invited to partake in the iPlay study. A total of 40 primary schools (consisting of 2208 10-12-yearold children) were eligible for participation, and were randomised to an intervention group or a control group. All 20 schools assigned to the control group formed the prospective cohort described in the present study, resulting in a cohort of 1091 10-12-year-old children. The study was approved by the Medical Ethics Committee of the VU University Medical Center, Amsterdam, The Netherlands. Informed consent was given by each child's parent or guardian by means of a passive informed consent.

\section{Design and measurements}

At the start of the school year (September 2006) all children completed a questionnaire in the classroom under the supervision of the researchers. The questionnaire collected information on demographic variables, as well as current sports and leisure time PA participation. This questionnaire was repeated at the end of the school year (May 2007).

Exposure to PE classes was equal in all schools (ie, twice a week for $45 \mathrm{~min}$ ). The weekly individual exposure of 90 min was multiplied by the number of weeks between baseline and followup, taking the regular school holidays into account.

Individual exposure to sports and leisure time PA was derived from the baseline and follow-up questionnaires. Both questionnaires contained questions on the weekly frequency and duration of sports and leisure time PA, from which weekly exposure was estimated. Baseline or follow-up data required to calculate weekly leisure time PA 
exposure was missing for 35 children. These children were removed from further analyses concerning overall injuries, as well as leisure time PA-related injuries. There were no missing data regarding exposure to sports participation.

Mean weekly sports and leisure time PA exposure was calculated from the baseline and follow-up weekly exposure values, and multiplied by the number of weeks between the completion of the questionnaires. A correction factor of 0.8 was used in order to account for seasonal effects on PA participation throughout the follow-up period. Although chosen arbitrarily, this correctional factor is in line with the decrease in PA during winter as found in previous studies. ${ }^{11}$

Throughout the follow-up period PA injuries were continuously monitored by PE teachers. In case of injury the PE teacher provided the injured child with an injury registration form, which had to be completed within 7 days of injury onset, preferably with help from the PE teacher. On this form the child was asked to provide information on the injury location, injury type, injury diagnosis, direct cause of the injury, subsequent medical treatment and activity performed at time of injury (ie, PE class, leisure time PA, or sports).

\section{Injury definition}

An injury was recorded if it occurred during either PE class, leisure time PA, or sports, and caused the child: ${ }^{8}$

- to stop his/her current activity; and/or

- to not (fully) participate in the next planned physical activity (applicable as well to regular leisure time PA); and/ or

- to be unable to go to school the next day; and/or

- to seek medical attention (ranging from onsite care by eg, first aid personnel, to personal care by eg, physiotherapist or sports doctor).

\section{Analysis}

Injury incidence density (ID) and corresponding 95\% confidence intervals $(95 \% \mathrm{CI})$ were calculated for all participants, as well as for boys and girls separately, as the number of new injuries reported per $1000 \mathrm{~h}$ of exposure. Therefore, exposure time of each individual child until the onset of first injury within each specific modality of PA was used.

For boys compared to girls, as well as between the different modalities of PA, incidence density ratios (IDRs) and corresponding 95\% CIs were calculated. Differences in sports and school absence due to injury were analysed using $\chi^{2}$ statistics.

\section{RESULTS}

\section{Research population}

Of the 20 schools that agreed to participate in the study, all completed the entire follow-up period. One or more questionnaires were missing for 95 individual participating children. Consequently, these children were excluded from the analyses. This resulted in a sample of 996 children (493 boys and 503 girls) (table 1).

\section{Injury incidence density}

During the follow-up period a total of 119 injuries were reported by 104 children. This resulted in an overall injury ID of 0.48 per $1000 \mathrm{~h}$ of exposure (95\% CI 0.38 to 0.57 ) (table 2). In general the injury ID was lowest for leisure time PA $(0.39 ; 95 \%$ CI 0.28 to 0.50$)$, followed subsequently by PE $(0.50 ; 95 \%$ CI 0.29 to $0.71)$ and sports $(0.66 ; 95 \%$ CI 0.46 to 0.87$)$. Injury ID did not differ significantly between the different modalities of PA. A
Table 1 Participant characteristics

\begin{tabular}{lccc}
\hline & Total population & \multicolumn{1}{l}{ Boys } & \multicolumn{1}{l}{ Girls } \\
\hline $\mathrm{n}$ & 996 & $493(49.5 \%)$ & $503(50.5 \%)$ \\
Age, years & $10.7(0.8)$ & $10.7(0.8)$ & $10.6(0.8)$ \\
Height, cm & $149.1(7.9)$ & $148.7(7.6)$ & $149.5(8.2)$ \\
Weight, kg & $40.5(9.1)$ & $40.1(8.8)$ & $40.9(9.4)$ \\
Sports experience: & & & \\
None & $261(26.4 \%)$ & $110(22.4 \%)$ & $151(30.3 \%)$ \\
$<2$ years & $176(17.8 \%)$ & $78(15.9 \%)$ & $98(19.7 \%)$ \\
$2-4$ years & $259(26.2 \%)$ & $124(25.3 \%)$ & $135(27.1 \%)$ \\
$>4$ years & $292(29.6 \%)$ & $178(36.3 \%)$ & $114(22.9 \%$ \\
Missing & 8 & 3 & 5 \\
PA level, h/week: & & & \\
Overall & $7.5(2.9)$ & $8.1(2.9)$ & $7.0(2.7)$ \\
PE classes & $1.5(0.0)$ & $1.5(0.0)$ & $1.5(0.0)$ \\
Leisure time PA & $4.0(2.0)$ & $4.4(2.0)$ & $3.7(2.0)$ \\
Sports* & $2.0(1.7)$ & $2.2(1.7)$ & $1.7(1.6)$ \\
\hline
\end{tabular}

Data are given as mean (SD) or $n,(\%)$ of the entire population (\%).

* Mean hours of sports participation per week has been calculated taken only into account that part of the population that reported to participate in organised sports activities.

PA, physical activity; PE, physical exercise.

similar distribution was found for boys and girls separately. However, for boys the injury ID due to sports was significantly higher than for leisure time PA (IDR 2.61; 95\% CI 1.39 to 4.95). In general for girls a higher injury ID was reported than for boys (IDR $1.62 ; 95 \%$ CI 1.10 to 2.39 ), mainly due to girls having a more than twofold higher injury ID resulting from leisure time PA (IDR 2.13; 95\% CI 1.18 to 3.84 ).

Of all injuries $40 \%$ required medical treatment, resulting in an incidence of 0.19 medically treated injuries per $1000 \mathrm{~h}$ of exposure (95\% CI 0.13 to 0.25 ). The incidence of medically treated injuries was divided equally between the different modalities of PA, as well as between genders.

\section{Nature and cause of injuries}

Figure 1 depicts the anatomical location of injuries divided across the different modalities of PA. In general most injuries were to the lower extremities (68\%), of which the majority $(42 \%)$ were to the lower leg or ankle. Injuries to the lower leg or ankle were predominantly reported resulting from sports, while injuries to the lower arm, hand or wrist were mostly reported to be due to leisure time PA.

Bruises were most commonly reported (43\%), mainly occurring during leisure time PA (fig 2). The second most frequent occurring injury was a ligament sprain, most commonly resulting from participation in sports.

Falling or stumbling as well as missteps were most common, each accounting for $28 \%$ of all injuries (fig 3). Collisions with materials (eg, a ball, playground obstacle, etc) accounted for a substantial amount of injury causes as well (22\% of all injuries). During leisure time PA falling or stumbling and collision with materials accounted for most injuries and $45 \%$ and $29 \%$ of all leisure time PA activities, respectively. In sports most injuries were caused by a misstep or twisting motion (41\% of all sports injuries).

\section{Sports and school absence}

Within the injuries sustained by children who reported to participate in organised sports, 70 injuries (68\%) resulted in absence from sports participation due to the injury. For boys $(\mathrm{n}=37 ; 79 \%)$ the number of injuries resulting in absence from sports was, although not significant, higher than for girls 


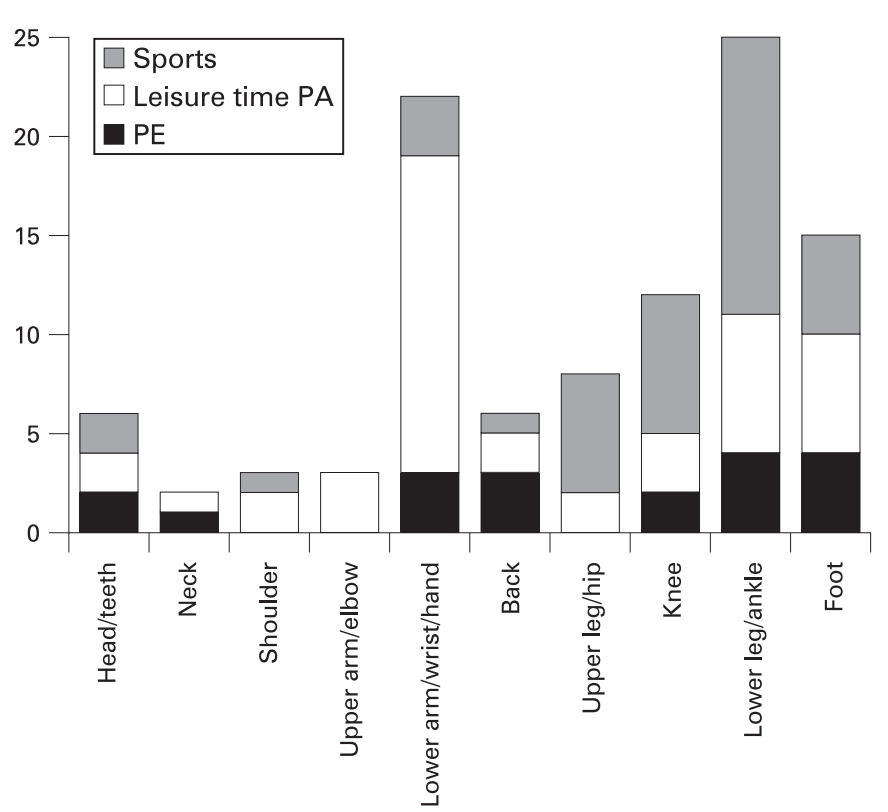

Figure 1 Injury location within the different modalities of physical activity (PA), presented as the percentage of all injuries.

( $n=33 ; 59 \%$ ). A sports absence of 1 week was reported in $45 \%$ of all cases of injury, 29\% resulted in an absence of 2 weeks and $26 \%$ resulted in an absence of 3 weeks or more. The duration of absence was similarly divided between boys and girls.

Absence from regular school activities was reported in $14 \%$ $(n=17)$ of all injuries, with a mean loss of 1.8 school days per injury (SD 1.3). Reported absence from school due to injury and mean loss of school days was similar for boys (14\%; 1.7 (SD 1.0) days lost) and girls (15\%; 1.9 (SD 1.6) days lost).

\section{DISCUSSION}

This prospective study carried out during 1 school year showed an overall injury ID of 0.48 injuries per $1000 \mathrm{~h}$ of PA. Injury ID was lowest for leisure time PA, followed by PE and sports, respectively.

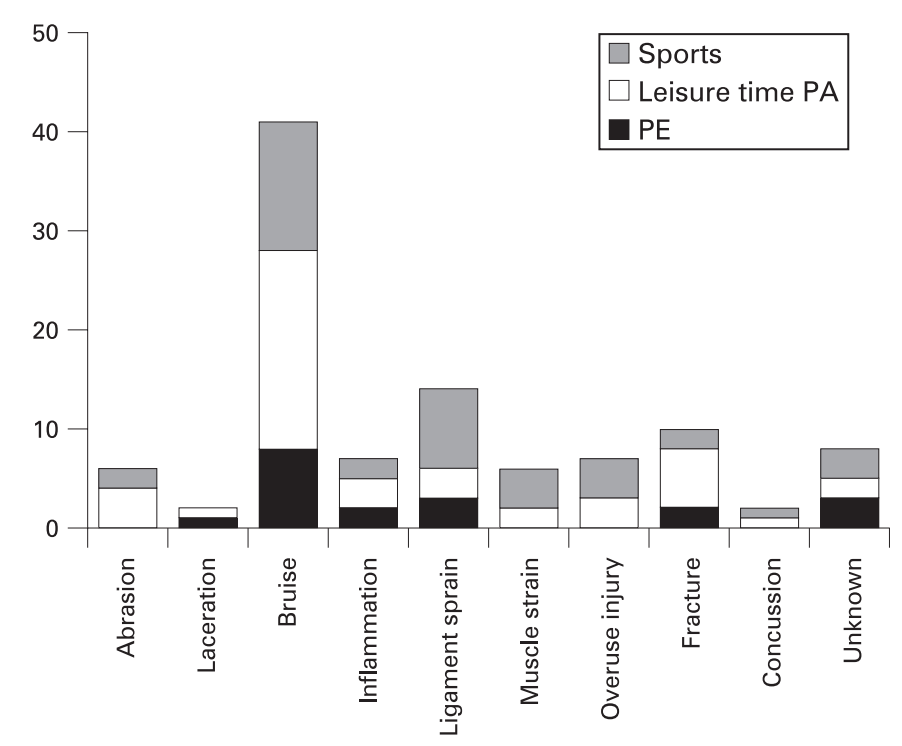

Figure 2 Injury type within the different modalities of physical activity (PA), presented as the percentage of all injuries. 


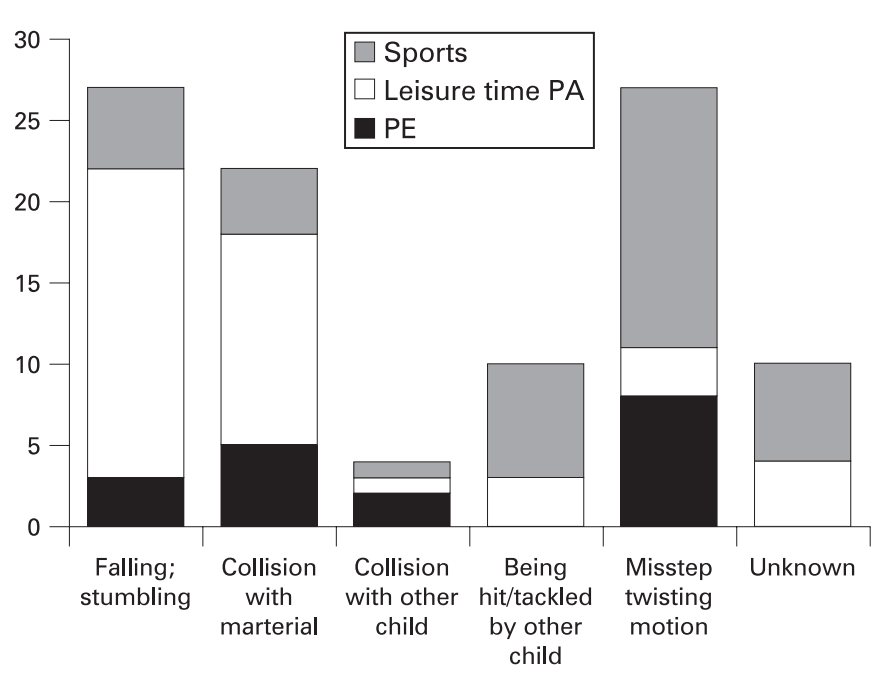

Figure 3 Injury mechanisms within the different modalities of physical activity (PA), presented as the percentage of all injuries.

As in most descriptive studies, comparisons of incidence figures are hampered by differences in study design, population and injury definition. When it comes to sports and PA injuries in children a proper comparison is further complicated by the small number of available studies. As indicated by Spinks and McClure, ${ }^{12}$ injury studies in young children focus mostly on sports-specific injury risk. Our study specifically dealt with injuries in a relative young age group of 10-12-year-old children, and investigated the entire scope of PA modalities these children may participate in. Furthermore, the majority of studies report on injuries sustained by a wide age range of children. However, as found by Sorensen et $a l^{13}$ great variations in sports and PA injury rates exist between different ages, ranging from 20 per 1000 children at the age of 6 , to 120 per 1000 children at the age of 14 . The present study included only a relative small age range of 10-12-year-old children, rendering a comparison with other studies unhelpful. The only study comparable in design and methodology is by Spinks et al. ${ }^{14}$ For the same age group they reported similar overall injury rates of 0.59 injuries per $1000 \mathrm{~h}$ sports and PA participation, as well as 0.69 sports injuries per $1000 \mathrm{~h}$ of sports participation.

In contrast to what is generally being reported in the literature, we found the overall injury ID of girls to be higher than for boys. Corresponding to our study, Sundblad et al ${ }^{15}$ reported higher $\mathrm{PE}$ injury rates for girls as compared to boys. For other modalities of PA no differences between genders were found. This latter contrasts to our result that girls have a twofold injury risk during leisure time PA. It may well be that the definition of leisure time PA differs between studies. It remains unclear in the study of Sundblad et $a l^{15}$ what was defined as leisure time PA. Even though the present study is the first to observe these gender differences in such a pronounced way, Sorensen et al ${ }^{13}$ previously indicated that gender differences in injury risks "crossover" between the ages 12-14. This is presumably due to the growth spurt appearing earlier in girls. It was only in the older age groups that Sorensen et a ${ }^{13}$ showed injury risk to be substantially higher in boys.

Of all injuries, $40.3 \%$ required some form of medical attention and $68 \%$ resulted in absence from sports or school. This is a grave amount, especially when one considers the broad injury definition used (ie, any occurrence that led at least to cessation of the current activity). Although this has led to the registration of a substantial amount of relatively "minor" injuries (eg, 43\% of all reported injuries were bruises), this shows that an important number of injuries in children should be labelled as "serious" or even "severe", especially in the light of the young, growing and playing child. This high percentage of serious injuries warrants a stronger focus on injury preventive efforts in younger children.

Another methodological issue that needs to be addressed is the registration of participation in leisure time PA and sports. In contrast to participation in PE classes, individual exposure to sports and leisure time PA was estimated from self-reported weekly frequency and duration of sports and leisure time PA, in the baseline and follow-up questionnaires. PA and sports participation would have been more accurate when measured objectively and on a more regular basis (eg, weekly, using structured exposure forms commonly used in sports injury research). ${ }^{16}$ However, from a practical perspective this was unachievable, and hence we chose a method that might have resulted in a slight overestimation of actual exposure to leisure time PA and sports. Despite this potential bias we were able to calculate injury ID per $1000 \mathrm{~h}$ of exposure, giving an estimation of actual differences in injury risk between the different modalities of PA.

An important strength of this study was that all PA-related injuries of each individual child were prospectively registered on a weekly basis by the PE teacher. Therefore, we believe that close to all injuries resulting from participation in $\mathrm{PE}$, leisure time PA and sports have been reported. In addition, the injury definition used was not limited to injuries resulting in at least absence from school or sports, or injuries requiring medical treatment. Both are injury definitions widely used in (sports) injury research in children. ${ }^{12-15}{ }^{16-20}$ Such definitions may lead to an underestimation of the actual number of injuries, the socalled "tip of the iceberg" phenomenon, as only more "severe" injuries are being registered. ${ }^{21}$ This showed, for instance, in the high number of bruises found in the present study; injuries rarely causing time loss from PE classes or sports, but potentially hampering vitally important leisure time PA for the child.

\section{Conclusions}

An important purpose of descriptive studies such as the present work is to supply knowledge on the magnitude, severity and aetiology of injuries in order to provide a basis for preventive measures. Concerning 10-12-year-old children, descriptive information on sports and PA injuries is lacking, although a substantial sports and PA injury risk exists in this age group. In general injury ID was found to be highest for participation in organised sports, where most injuries were caused by missteps or twisting motions leading to ligament injuries to the lower extremities. The most common injuries were bruises, most frequently leading to upper extremity injuries through falling and stumbling during leisure time PA. Next to such specific areas of preventive interest it was found that in this specific age group girls require special attention as they seem to be at higher injury risk than boys.

Funding: This study was funded by The Netherlands Organisation for Health Research and Development, grant number 62200033.

Competing interests: None.

Ethics approval: This study was approved by the Medical Ethics Committee of the VU University Medical Center, Amsterdam, The Netherlands.

\section{REFERENCES}

1. Ekblom B, Astrand PO. Role of physical activity on health in children and adolescents. Acta Paediatrica 2000;89:762-4. 
2. Hallal PC, Victora CG, Azevedo MR, et al. Adolescent physical activity and health: a systematic review. Sports Med 2006;36:1019-30.

3. Parkkari J, Kujala UM, Kannus P. Is it possible to prevent sports injuries? Review of controlled clinical trials and recommendations for future work. Sports Med 2001;31:985-95.

4. Adirim TA, Cheng TL. Overview of injuries in the young athlete. Sports Med 2003;33:75-81.

5. Abernethy L, MacAuley D. Impact of school sports injury. Br J Sports Med 2003;37:354-5.

6. Flynn JM, Lou JE, Ganley TJ. Prevention of sports injuries in children. Curr Opin Pediatr 2002;14:719-22.

7. van Mechelen W, Verhagen EALM. Essay: injury prevention in young people - time to accept responsibility. Lancet 2005;366(Suppl 1):S46.

8. Van Mechelen W, Hlobil H, Kemper HCG. Incidence, severity, aetiology and prevention of sports injuries. A review of concepts. Sports Med 1992;14:82-99.

9. Spinks AB, McClure RJ. Quantifying the risk of sports injury: a systematic review of activity-specific rates for children under 16 years of age. Br J Sports Med 2007:41:548-57.

10. Collard DCM, Chin A Paw M, van Mechelen W, et al. Design of the iPlay study: systematic development of a physical activity injury prevention program for primary school children. Sports Med. In press.

11. Tucker $\mathbf{P}$, Gilliland J. The effect of season and weather on physical activity: a systematic review. Pub Health 2007;121:909-22.
12. Spinks $\mathbf{A B}$, McClure RJ. Quantifying the risk of sports injury: a systematic review of activity-specific rates for children under 16 years of age. Br J Sports Med 2007; 41:548-57.

13. Sorensen L, Larsen SE, Rock ND. The epidemiology of sports injuires in school-aged children. Scand J Med Sci Sports 1996;6:281-6.

14. Spinks AB, McClure RJ, Bain C, et al. Quantifying the association between physical activity and injury in primary school-aged children. Pediatrics 2006;118:e43-50.

15. Sundblad G, Saartok T, Engstrom L, et al. Injuries during physical activity in school children. Scand J Med Sci Sports 2005;15:313-23.

16. Currie $\mathbf{C E}$, Williams $\mathbf{J} \mathrm{M}$, Wright $\mathrm{P}$, et al. Incidence and distribution of injury among schoolchildren aged 11-15. Inj Prev 1996;2:21-5.

17. de Loës M. Epidemiology of sports injuries in the Swiss organization "Youth and Sports" 1987-1989. Injuries, exposure and risks of main diagnoses. Int J Sports Med 1995; 16:134-8.

18. Lowry R, Lee SM, Galuska DA, et al. Physical activity-related injury and body mass index among US high school students. J Phys Act Health 2007:4:325-42.

19. Backx FJG, Erich WBM, Kemper ABA, et al. Sports injuries in school-aged children. An epidemiologic study. Am J Sports Med 1989;17:234-9.

20. Janssen I, Dostaler S, Boyce WF, et al. Influence of multiple risk behaviors on physical activity related injuries in adolescents. Pediatrics 2007;119:e673-80.

21. Walter S, Sutton J, Mclntosh J, et al. The aetiology of sports injuries. A review of methodologies. Sports Med 1985;2:47-58. 


\title{
BJSM
}

\section{A prospective cohort study on physical activity and sports-related injuries in 10 - 12-year-old children}

\author{
E Verhagen, D Collard, M Chin A Paw, et al.
}

Br J Sports Med 2009 43: 1031-1035 originally published online January 5, 2009

doi: 10.1136/bjsm.2008.055483

Updated information and services can be found at:

http://bjsm.bmj.com/content/43/13/1031.full.html

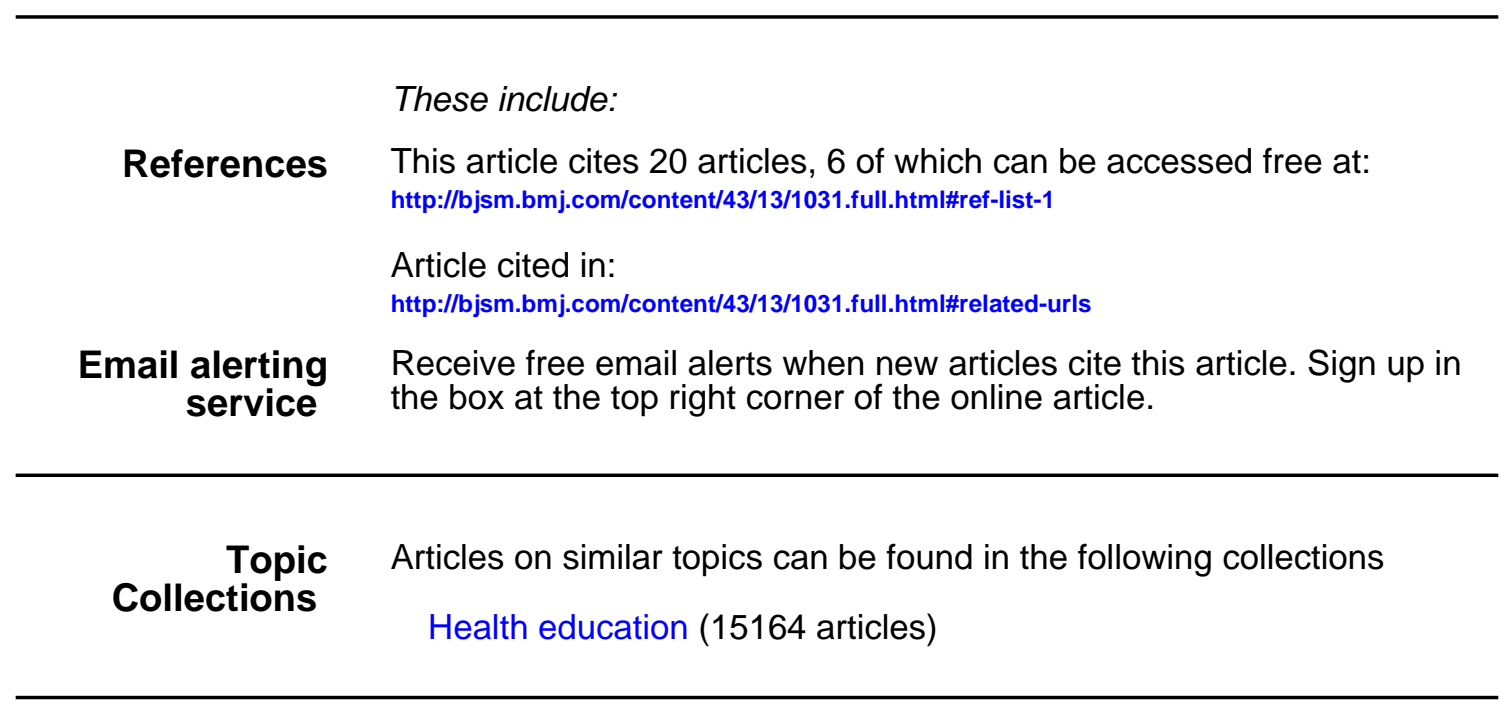

Notes

To request permissions go to:

http://group.bmj.com/group/rights-licensing/permissions

To order reprints go to:

http://journals.bmj.com/cgi/reprintform

To subscribe to BMJ go to:

http://group.bmj.com/subscribe/ 